\title{
Changes in right heart haemodynamics and echocardiographic function in an advanced phenotype of pulmonary hypertension and right heart dysfunction associated with pulmonary fibrosis
}

\author{
Rajeev Saggar, ${ }^{1}$ Dinesh Khanna, ${ }^{2}$ Anjali Vaidya, ${ }^{3}$ Ariss Derhovanessian, ${ }^{4}$ \\ Paul Maranian, ${ }^{2}$ Erin Duffy, ${ }^{5}$ John A Belperio, ${ }^{4}$ Sam S Weigt, ${ }^{4}$ Shiv Dua, ${ }^{6}$ \\ Shelley S Shapiro, ${ }^{4}$ Jonathan G Goldin, ${ }^{7}$ Fereidoun Abtin, ${ }^{7}$ Joseph P LynchIII, ${ }^{4}$ \\ David J Ross, ${ }^{4}$ Paul R Forfia, ${ }^{8}$ Rajan Saggar ${ }^{4}$
}

- Additional material is published online only. To view please visit the journal online (http://dx.doi.org/10.1136/ thoraxjnl-2013-204150).

For numbered affiliations see end of article.

\section{Correspondence to} Dr Rajan Saggar, David Geffen School of Medicine, University of California Los Angeles, 10833 Le Conte Ave. CHS 37-131, Los Angeles, CA 90095, USA; rsaggar@mednet.ucla.edu

Received 8 July 2013 Revised 19 December 2013 Accepted 20 December 2013

\section{SLinked}

- http://dx.doi.org/10.1136/ thoraxjnl-2013-204964

CrossMark

To cite: Saggar $\mathrm{R}$,

Khanna D, Vaidya A, et al.

Thorax 2014;69:123-129.

\section{ABSTRACT}

Background Pulmonary hypertension (PH)-targeted therapy in the setting of pulmonary fibrosis (PF) is controversial; the main clinical concern is worsening of systemic hypoxaemia. We sought to determine the effects of gentle initiation and chronic administration of parenteral treprostinil on right heart function in patients with PF associated with an advanced PH phenotype.

Methods Open-label, prospective analysis of patients with PF-PH referred for lung transplantation (LT). Advanced PH was defined as mean pulmonary artery pressure (mPAP) $\geq 35 \mathrm{~mm} \mathrm{Hg}$. We compared haemodynamics, Doppler echocardiography (DE), oxygenation, dyspnoea and quality of life indices, and 6 min walk distance (6MWD) before and 12 weeks after parenteral treprostinil.

Results 15 patients were recruited in the study. After therapy, there were significant improvements in right heart haemodynamics (right atrial pressure ( $9.5 \pm 3.4$ vs $6.0 \pm$ 3.7); mPAP ( $47 \pm 8$ vs $38.9 \pm 13.4) ; \mathrm{Cl}(2.3 \pm 0.5$ vs $2.7 \pm$ 0.6); pulmonary vascular resistance ( $698 \pm 278$ vs $496 \pm$ 229); transpulmonary gradient ( $34.7 \pm 8.7$ vs $28.5 \pm 10.3)$; $\mathrm{mvO}_{2}(65 \pm 7.2$ vs $70.9 \pm 7.4)$; and stroke volume index (29.2 \pm 6.7 vs $33 \pm 7.3))$ and DE parameters reflecting right heart function (right ventricular (RV) end diastolic area $\left(36.4 \pm 5.2\right.$ vs $\left.30.9 \pm 8.2 \mathrm{~cm}^{2}\right)$, left ventricular eccentricity index (1.7 \pm 0.6 vs $1.3 \pm 0.5)$, tricuspid annular planar systolic excursion $(1.6 \pm 0.5$ vs $1.9 \pm 0.2 \mathrm{~cm})$ ). These changes occurred without significant alteration in systemic oxygenation, heart rate, or mean systemic arterial pressure. In addition, improvements were seen in 6MWD (171 \pm 93 vs $230 \pm 114$ ), 36-Item Short Form Health Survey Mental Component Summary aggregate ( $38 \pm 11$ vs $44.2 \pm 10.7$ ), University of California, San Diego Shortness of Breath Questionnaire $(87 \pm 17.1$ vs $73.1 \pm 21)$, and brain natriuretic peptide ( $558 \pm 859$ vs $228 \pm 340$ ).

Conclusions $\mathrm{PH}$-targeted therapy may improve right heart haemodynamics and echocardiographic function without affecting systemic oxygen saturation in an advanced PH phenotype associated with RV dysfunction in the setting of PF.

\section{INTRODUCTION}

Pulmonary hypertension (PH) may complicate pulmonary fibrosis $(\mathrm{PF})$ of different causes, but few

\section{Key messages}

What is the key question?

- What are the effects of parenteral treprostinil on right heart function of patients with pulmonary fibrosis (PF) referred for lung transplantation (LT) with an advanced pulmonary hypertension $(\mathrm{PH})$ phenotype?

What is the bottom line?

- Parenteral treprostinil improves right heart haemodynamics and echocardiographic function without affecting systemic oxygen saturation in PH-PF with an advanced PH phenotype.

Why read on?

- This pilot study suggests parenteral treprostinil improves right ventricle function and is a safe therapeutic option in patients with PH-PF with an advanced PH phenotype; as such, this approach may be a consideration for patients with advanced PH-PF if they are ineligible for, or as a bridge to, LT.

studies have focused on the treatment of advanced $\mathrm{PH}$ in this context. ${ }^{1-4}$ Furthermore, no prospective chronic parenteral prostanoid administration studies are available in patients with PF homogenised for the less common advanced PH phenotype, characterised by significantly altered right heart haemodynamics and right ventricular (RV) dysfunction. We previously reported a case where the rationale for parenteral treprostinil as a bridge to lung transplantation (LT) was outlined in the index patient with PF-PH for this study. ${ }^{5}$ Importantly, parenteral prostanoid therapy associated with worsening of ventilation-perfusion (V-Q) mismatch and subsequent hypoxaemia remains a major clinical concern. The purpose of this pilot study was to evaluate the effects of acute and subsequent chronic parenteral treprostinil therapy on right heart haemodynamics and echocardiographic function in patients with $\mathrm{PH}$ referred for LT in the setting of an advanced $\mathrm{PH}$ phenotype and right heart dysfunction. 


\section{MATERIALS AND METHODS}

This study was approved by the IRB at University of California, Los Angeles (UCLA IRB\# 07-11-087-03; clinicaltrials.gov Identifier NCT00705133). We recruited 15 outpatients with PF referred to our LT programme between July 2008 and January 2011 who had advanced PH based on right heart catheterisation (RHC) (figure 1). Sarcoidosis and systemic sclerosis spectrum of disease were excluded, as were patients requiring $>10 \mathrm{~L} / \mathrm{min}$ of oxygen at baseline. Importantly, threshold measures of right heart size and/or function were not required for study enrolment. Patients with combined pulmonary fibrosis emphysema were included. ${ }^{6}$ Formal pulmonary rehabilitation was not prescribed during the study period.

Advanced $\mathrm{PH}$ was defined using haemodynamic criteria: mean pulmonary artery pressure $(\mathrm{mPAP}) \geq 35 \mathrm{~mm} \mathrm{Hg}$, pulmonary artery wedge pressure (PAWP) $\leq 15 \mathrm{~mm} \mathrm{Hg}$, and pulmonary vascular resistance $(\mathrm{PVR})>240 \mathrm{dyn} \mathrm{s} / \mathrm{cm}^{5}$. Other $\mathrm{PH}$ aetiologies were ruled out based on current recommendations. ${ }^{7}$ All follow-up RHCs were performed after $\geq 12$ weeks of stable dose background PH-targeted therapy, and background PF-related therapy remained unaltered during the study period.

\section{Six minute walk distance and oxygen supplementation protocols}

Patients were ambulated per American Thoracic Society criteria with a modification regarding oxygen supplementation (OS), given the inherent hypoxaemia in this patient population (see online supplementary repository). ${ }^{8}$

\section{Pulmonary function testing and Doppler echocardiogram protocols}

Pulmonary function testing (PFT) was obtained at treprostinil initiation and at 12 weeks and included forced vital capacity (FVC), forced expiratory volume in $1 \mathrm{~s}\left(\mathrm{FEV}_{1}\right), \mathrm{FEV}_{1} / \mathrm{FVC}$ ratio and single-breath diffusing capacity for carbon monoxide
(DLCO). Total lung capacity (TLC) was only performed at baseline. Doppler echocardiogram (DE) was performed using conventional equipment (Hewlett-Packard, Palo Alto, California, USA) at baseline and 12 weeks (see online supplementary repository).

\section{Dyspnoea and quality of life assessments}

Dyspnoea was measured with the University of California, San Diego Shortness of Breath (UCSD SOB) questionnaire and the Borg Dyspnoea Index (BDI) (see online supplementary repository).

Health-related quality of life was measured with the 36-Item Short Form Health Survey (SF-36). SF-36 scales are summarised into Physical Component (PCS) and Mental Component (MCS) Summary scores. The eight SF-36 scales and the PCS and MCS scores are standardised to a mean of 50 and an SD of 10 in the general US population. Minimally important difference (MID) estimates for SF-36 PCS and MCS are 2.5 points (see online supplementary repository).

\section{Parenteral treprostinil titration protocol}

All patients were hospitalised for $48 \mathrm{~h}$ for treprostinil initiation and uptitration (see online supplementary repository).

\section{High-resolution CT lung parenchymal scoring}

Thin slice $(<3 \mathrm{~mm})$ CT scans were used for objective assessment of lung parenchymal abnormality at baseline (see online supplementary repository).

\section{Study parameters}

Patient assessments were made at baseline and after 12 weeks of parenteral treprostinil and included RHC and systemic haemodynamics, echocardiographic parameters, 6 min walk distance (6MWD), PFT, systemic and central oxygenation, brain natriuretic peptide (BNP), and SF-36/UCSD SOB questionnaires.

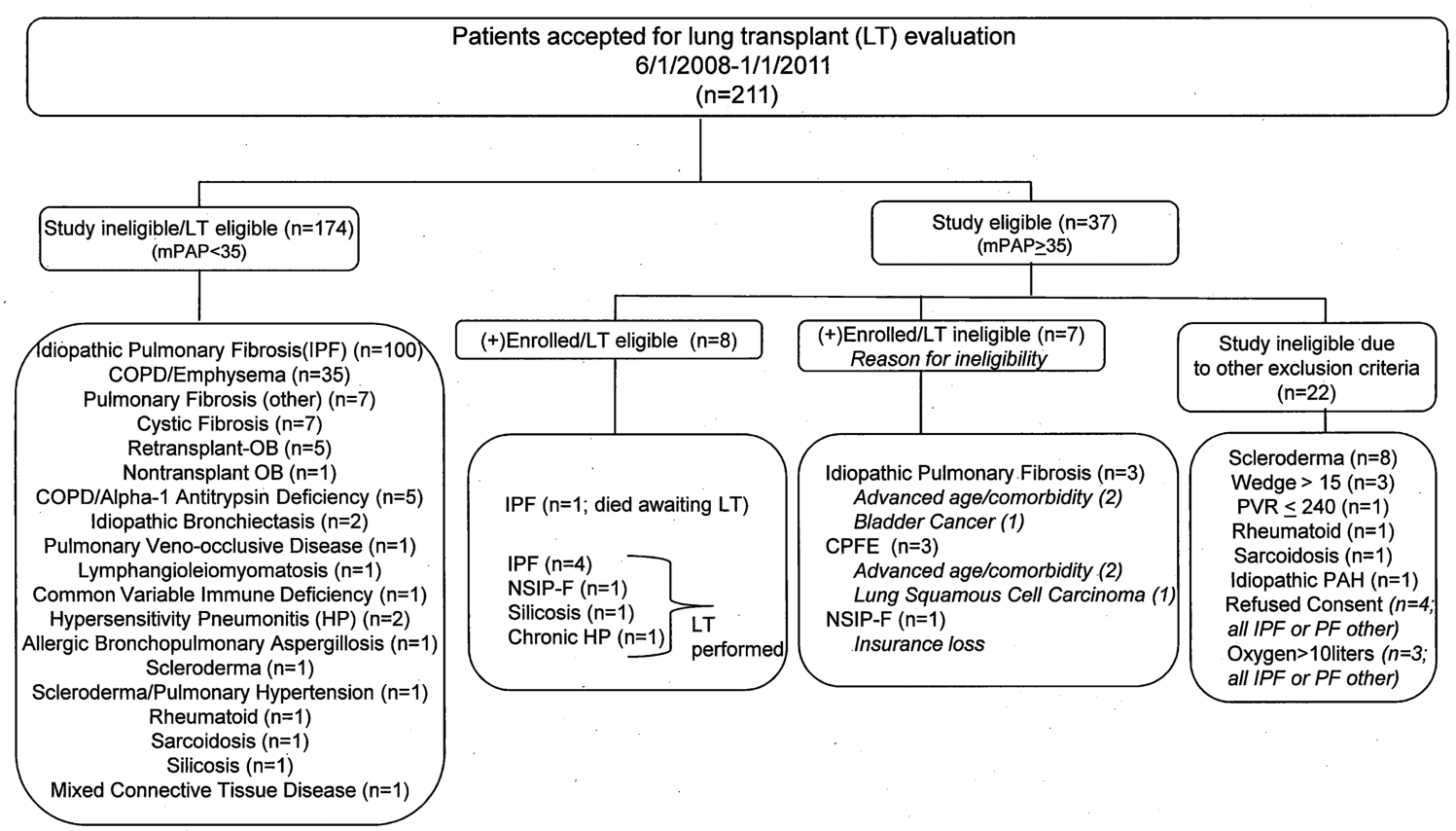

Figure 1 Recruitment of patients $(n=15)$ with pulmonary fibrosis and advanced pulmonary hypertension being evaluated for lung transplantation at a single tertiary medical centre between July 2008 and January 2011. CPFE, combined pulmonary fibrosis emphysema; mPAP, mean pulmonary artery pressure; NSIP-F, non-specific interstitial pneumonia fibrosis; PAH, pulmonary arterial hypertension; PF, pulmonary fibrosis; PVR, pulmonary vascular resistance. 


\section{Statistics}

Baseline characteristics were described as frequencies (\%) for categorical variables and means (SD) for continuous variables. Clinical, haemodynamic, and echocardiographic data were reported at baseline and 12 weeks, and the Shapiro-Wilk test was used to assess the normaility of the distributions of baseline and week 12 data. For variables where normality was rejected at the $\mathrm{p}<0.05$ level, median (IQR) values were reported and the Wilcoxon signed rank test was used to compare DLCO (\% predicted), FVC\%/DLCO\%, 6 min walk $10 \mathrm{~L}$ face mask (FM) (\% saturation), SF-36 Physical Functioning, stroke volume (SV), and systolic blood pressure (SBP) measures at baseline and week 12 . For all other clinical, haemodynamic, and echocardiographic variables, mean (SD) were reported and paired t tests were used to compare variables at baseline and week 12. Q-values were computed to assess the impact of multiple comparisons in the domains of pulmonary function, quality of life, and haemodynamics. Analyses were conducted with Stata V.13 (Stata Corp LP, College Station, Texas, USA), and $\mathrm{p}$ values $<0.05$ were considered statistically significant.

\section{RESULTS}

\section{Demographics}

Fifteen patients aged $63 \pm 15$ years (mean \pm SD) (20\% female) referred for LT met all study criteria and agreed to enrolment (figure 1). Baseline WHO functional class was equally split with $53 \%$ class III $(n=8)$ and $47 \%$ class IV $(n=7)$. Background PH therapy ( $\geq 12$ weeks of stable dose therapy) and the underlying clinical diagnoses regarding the aetiology of the PF are displayed in table 1. A subgroup of clinical diagnoses $(n=10)$ had pathological confirmation made by surgical lung biopsy $(n=2)$, eventual explantation $(n=7)$, or autopsy $(n=1)$ (see online

Table 1 Patient demographics, underlying fibrotic lung disease clinical subtype, and background $\mathrm{PH}$-targeted therapy

\begin{tabular}{|c|c|c|}
\hline \multirow[b]{2}{*}{ Patient characteristics } & \multicolumn{2}{|l|}{$\mathrm{N}=15$} \\
\hline & Mean & SD \\
\hline \multirow[t]{2}{*}{ Age in years } & 63 & 15 \\
\hline & $\mathbf{N}$ & Per cent \\
\hline \multicolumn{3}{|l|}{ NYHA class } \\
\hline III & 8 & 53 \\
\hline IV & 7 & 47 \\
\hline \multicolumn{3}{|l|}{ Race } \\
\hline Hispanic & 8 & 53 \\
\hline Caucasian & 4 & 27 \\
\hline Filipino/Japanese & 2 & 13 \\
\hline Middle Eastern & 1 & 7 \\
\hline \multicolumn{3}{|l|}{ Fibrotic lung disease clinical subtype } \\
\hline Idiopathic pulmonary fibrosis & 8 & 53 \\
\hline NSIP-fibrosis & 2 & 13 \\
\hline PF/emphysema (CPFE) & 3 & 20 \\
\hline Chronic Hypersensitivity Pneumonitis (HP) & 1 & 7 \\
\hline Silicosis & 1 & 7 \\
\hline \multicolumn{3}{|l|}{ Background therapy } \\
\hline Sildenafil monotherapy & 4 & 27 \\
\hline Bosentan monotherapy & 2 & 13 \\
\hline Sildenafil/bosentan combination & 3 & 20 \\
\hline None & 6 & 40 \\
\hline
\end{tabular}

supplementary repository table S2). Individual patient data are presented in the repository (see online supplementary table S1). The extent of baseline lung parenchymal abnormality by high-resolution CT (HRCT) chest imaging is reported for each patient in the repository (see online supplementary table S2).

\section{Safety/adverse events}

Of the 15 patients, 14 patients received subcutaneous treprostinil and 1 patient was placed on intravenous treprostinil. ${ }^{5}$ The treprostinil dose for the group at 12 weeks was $34 \pm 21 \mathrm{ng} / \mathrm{kg} /$ min $($ mean $\pm \mathrm{SD}$ ) and a range of $18-97 \mathrm{ng} / \mathrm{kg} / \mathrm{min}$. During inpatient treprostinil initiation, there were no changes in vital signs, particularly oxygen saturation by peripheral pulse oximetry (PPO), or adverse haemodynamic changes that led to acute discontinuation of the medication. During inpatient and outpatient treprostinil uptitration, patients experienced typical prostanoid effects, including jaw pain, diarrhoea, lower extremity bone pain, site pain/reaction, headache, and/or flushing. ${ }^{9}$

\section{Pulmonary function testing, 6MWD, and oxygen status}

The mean $( \pm \mathrm{SD})$ baseline $\%$ predicted values for $\mathrm{FEV}_{1}, \mathrm{FVC}$, TLC, and $\mathrm{FEV}_{1} / \mathrm{FVC}$ ratio were 62 (17), 62 (21), 70 (15), 77 (11), and 24 (13), respectively and the median (IQR) baseline \% predicted value for DLCO was 24 (13); for the cohort of patients without CPFE $(n=12)$, baseline TLC was 67 (16). There were no significant changes in PFT parameters following 12 weeks of treprostinil (table 2). Comprehensive individual patient data are presented in the repository (see online supplementary table S3).

The baseline 6MWD (mean \pm SD) was $171 \pm 93 \mathrm{~m}$ with a resting room air pulse oximetry of $83 \pm 7 \%$. All except one

Table 2 Pulmonary function testing, oxygen requirements, and 6 min walk distance with Borg Dyspnoea Index (BDI) scores at baseline and end of study

\begin{tabular}{|c|c|c|c|}
\hline & $\begin{array}{l}\text { Baseline } \\
\mathrm{N}=15 \\
\text { Mean (SD) }\end{array}$ & $\begin{array}{l}12 \text { weeks } \\
\mathrm{N}=15 \\
\text { Mean (SD) }\end{array}$ & p Value* \\
\hline \multicolumn{4}{|l|}{ Pulmonary function } \\
\hline FVC, \% predicted & $62(21)$ & $63(18)$ & 0.687 \\
\hline $\mathrm{FEV}_{1}, \%$ predicted & $62(17)$ & $64(16)$ & 0.215 \\
\hline $\mathrm{FEV}_{1} / \mathrm{FVC}$ & $77(11)$ & $80(12)$ & 0.134 \\
\hline \multicolumn{4}{|l|}{ TLC, \% predicted } \\
\hline All patients & $70(15)$ & - & \\
\hline Patients without $\mathrm{CPFE}, \mathrm{n}=12$ & $67(16)$ & - & \\
\hline DLCO, \% predictedt & $24(13)$ & $22(11)$ & $0.206 t$ \\
\hline FVC\%/DLCO $\% \dagger$ & $2.5(2.4)$ & $3.0(1.6)$ & $0.625 t$ \\
\hline Oxygen flow (L/min) & $3.9(1.9)$ & $3.9(2.1)$ & $>0.999$ \\
\hline \multicolumn{4}{|l|}{6 min walk: } \\
\hline 6 min walk distance $(m)$ & $171(93)$ & $230(114)$ & $<0.001$ \\
\hline Room air \% saturation & $83(7)$ & $80(10)$ & 0.078 \\
\hline $10 \mathrm{~L}$ face mask, $\%$ saturationt & $98(3)$ & $99(4)$ & $0.372 \dagger$ \\
\hline 10 L face mask, \% saturation nadir & $85(9)$ & $82(10)$ & 0.084 \\
\hline BDI score & $13.7(2.3)$ & $13.1(2.6)$ & 0.203 \\
\hline
\end{tabular}


patient required oxygen supplementation at rest; 11 of 15 (73\%) required $\geq 3 \mathrm{~L}$ of oxygen supplementation at rest. Table 2 shows the 6MWD improvements following 12 weeks of parenteral treprostinil therapy (mean $59 \mathrm{~m} ; \mathrm{p}<.001$ ). Specifically, 8 of 15 patients improved by $\geq 57 \mathrm{~m}$ (range $57-$ 150); 5 improved by $\geq 17 \mathrm{~m}$ (range 17-30); and 2 patients each declined by $10 \mathrm{~m}$ (figure 2). At 12 weeks, there were no significant differences in baseline oxygen requirements (see online supplementary repository figure S1) or oxygenation parameters by PPO either at rest on room air, at rest on $10 \mathrm{~L}$ FM, or at the completion of the $6 \mathrm{MWD}$ test on $10 \mathrm{~L} \mathrm{FM}$ (table 2). Individual patient data are presented in the repository (see online supplementary table S3).

\section{SF-36, UCSD SOB and BDI assessments}

Patients had a statistically significant improvement in UCSD SOB and SF-36 MCS scores at the end of the study $(\mathrm{p}<0.05$; table 3). When we assessed the clinical significance of these improvements, $77 \%$ and $50 \%$ had improvement $\geq$ MID estimates for UCSD SOB and SF-36 MCS, respectively. Additionally, there was no significant change in SF-36 PCS and BDI (table 3) between baseline and 12 weeks. Individual patient data are presented in the repository (see online supplementary table S4).

\section{Haemodynamics, BNP and Doppler echocardiography}

All 15 patients had baseline mPAP $\geq 35 \mathrm{~mm} \mathrm{Hg} ; 10$ (66\%) had $\mathrm{mPAP} \geq 40 \mathrm{~mm} \mathrm{Hg}$ and 7 (47\%) had $\mathrm{mPAP} \geq 50 \mathrm{~mm} \mathrm{Hg}$. The transpulmonary gradient (TPG) at baseline was $\geq 20 \mathrm{~mm} \mathrm{Hg}$ for all patients and $\geq 30 \mathrm{~mm} \mathrm{Hg}$ in 12 of 15 (80\%) patients. The average PVR was $698 \pm 278$ dyn $\mathrm{s} / \mathrm{cm}^{5}$, representing $44 \%$ of

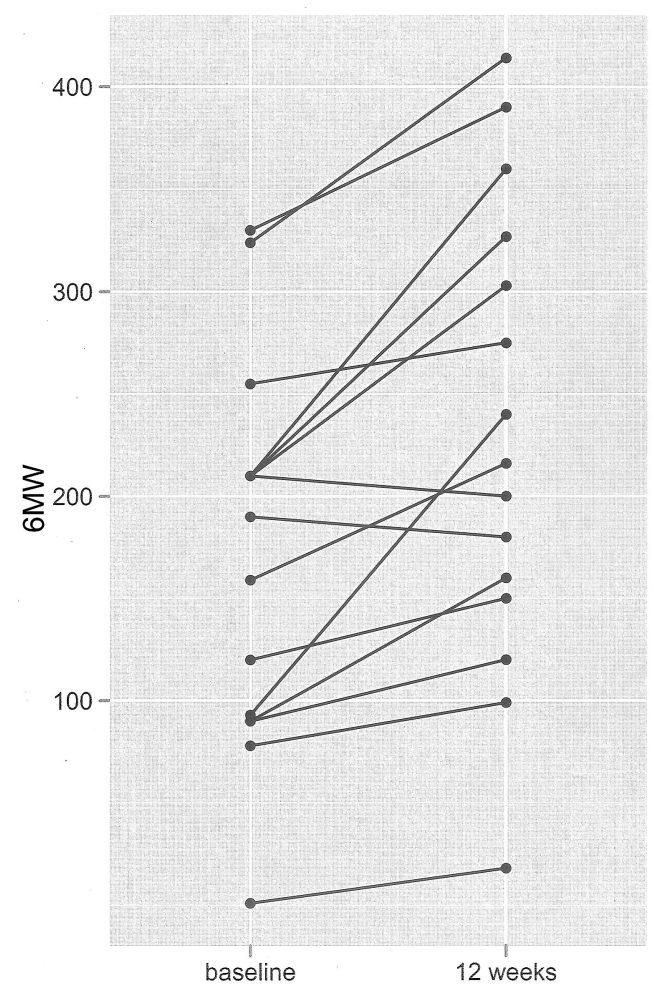

Figure 2 Individual 6 minute walk (6MW) distance responses to parenteral treprostinil at baseline and 12 weeks.
Table 3 Quality of life and dyspnoea score changes using Short Form 36 (SF-36) and University of California San Diego Shortness of Breath (UCSD SOB) questionnaire

\begin{tabular}{llll}
\hline & $\begin{array}{l}\text { Baseline } \\
\mathrm{N}=15 \\
\text { Mean (SD) }\end{array}$ & $\begin{array}{l}12 \text { weeks } \\
\mathrm{N}=15 \\
\text { Mean (SD) }\end{array}$ & p Value* \\
\hline UCSD SOB & $87(17.1)$ & $73.1(21)$ & 0.002 \\
SF-36 PCS aggregate & $27.1(5.8)$ & $28(8.8)$ & 0.479 \\
SF-36 MCS aggregate & $38(11)$ & $44.2(10.7)$ & 0.005 \\
Individual SF-36 domains & & & \\
Physical functioningt & $10.0(15.0)$ & $25.0(30.0)$ & $0.003 \dagger$ \\
Role-physical & $22.4(6.6)$ & $28.6(9.4)$ & 0.024 \\
Bodily pain & $47.6(11.1)$ & $39.4(9.4)$ & 0.049 \\
General health & $28.4(7.6)$ & $30.8(7.6)$ & 0.173 \\
Vitality & $36.9(10.4)$ & $41.9(9.1)$ & 0.026 \\
Social functioning & $28.0(9.4)$ & $33.9(13.6)$ & 0.014 \\
Role-emotional & $25.9(13.2)$ & $34.4(11.1)$ & 0.006 \\
Mental health & $42.4(10.8)$ & $45.3(9.6)$ & 0.150 \\
\hline
\end{tabular}

* Paired t test $p$ value presented, except when Wilcoxon signed rank indicated. †Data are non-normally distributed; median (IQR) and Wilcoxon signed rank $p$ value presented.

MCS, Mental Component Summary; PCS, Physical Component Summary.

the mean systemic vascular resistance (SVR). The baseline PVR was $\geq 480$ dyn s/cm ${ }^{5}$ in all but three patients. Following 12 weeks of treprostinil, there was evidence of decreased RV afterload, as per significant reductions in mPAP, TPG, PVR, and increased pulmonary capacitance (table 4; see online supplementary repository figure S2). Right heart function improved, as per reductions in right atrial pressure and increased mixed venous oxygen saturation, cardiac index and SV index (table 4). Although the SBP fell by $11 \mathrm{~mm} \mathrm{Hg}(124 \pm 21$ to $113 \pm$ $13 \mathrm{~mm} \mathrm{Hg} ; \mathrm{p}=0.028$ ), the mean systemic arterial pressure was not altered and there was a downward trend in the PVR/systemic vascular resistance $(S V R)$ ratio $(p=0.060)$, suggesting proportionally greater pulmonary than systemic vasodilation. Importantly, there were no significant changes in resting heart rate, arterial oxygen content, or oxygen delivery. Individual patient data are presented in the repository (see online supplementary table S5).

The two-dimensional echocardiographic examination at baseline revealed normal left ventricular cavity size (LV end-diastolic dimension $4.1 \pm 0.5 \mathrm{~cm}$ ) and systolic function (LV ejection fraction $64 \pm 6 \%$ ), and Doppler evidence of normal left atrial pressure (transmitral Doppler $\mathrm{E} / \mathrm{e}$ ' ratio $5.4 \pm 1.4$ ). In contrast, subjects were noted to have severe RV dilatation (RV enddiastolic area $36.4 \pm 5.2 \mathrm{~cm}^{2}$ ), marked right-to-left displacement of the interventricular septum (systolic eccentricity index $1.7 \pm$ 0.6) and moderate RV systolic dysfunction (tricuspid annular planar systolic excursion (TAPSE) $1.6 \pm 0.5 \mathrm{~cm}$; see online supplementary repository figure S3). Doppler estimated pulmonary artery systolic pressure at baseline was $72 \pm 12 \mathrm{~mm} \mathrm{Hg}$. The RV outflow tract (RVOT) acceleration time was markedly reduced (66 $\pm 16 \mathrm{~ms}$ ) and $100 \%$ of subjects showed evidence of systolic flow deceleration or 'notching' of the RVOT Doppler envelope. Of note, $85 \%$ of subjects showed evidence of both a TAPSE $<2.0 \mathrm{~cm}$ and RVOT Doppler notching, consistent with afterload-dependent RV dysfunction at baseline.

Figure 3 illustrates that following 12 weeks of parenteral treprostinil treatment, there were significant reductions in RV size $(p=0.021)$, less evidence of interventricular septal flattening 
Table 4 Systemic and pulmonary haemodynamics and oxygenation at baseline compared with 12 weeks after parenteral treprostinil therapy

\begin{tabular}{|c|c|c|c|}
\hline & $\begin{array}{l}\text { Baseline } \\
\mathrm{N}=15 \\
\text { Mean (SD) }\end{array}$ & $\begin{array}{l}12 \text { weeks } \\
N=15 \\
\text { Mean (SD) }\end{array}$ & p Value \\
\hline \multicolumn{4}{|l|}{ Haemodynamics, $\mathrm{mm} \mathrm{Hg}$} \\
\hline Right atrial pressure & $9.5(3.4)$ & $6.0(3.7)$ & $<0.001$ \\
\hline Mean pulmonary pressure & $47.0(8.0)$ & $38.9(13.4)$ & 0.005 \\
\hline Pulmonary artery wedge pressure & $12.5(4.1)$ & $10.5(6.1)$ & 0.247 \\
\hline Cardiac output (L/min) & $4.3(1.1)$ & $4.9(1.1)$ & 0.042 \\
\hline Cardiac index $\left(\mathrm{L} / \mathrm{min} / \mathrm{m}^{2}\right)$ & $2.3(0.5)$ & $2.7(0.6)$ & 0.017 \\
\hline PVR (dyn s/cm ${ }^{5}$ ) & $698(278)$ & $496(229)$ & $<0.001$ \\
\hline Mixed venous $\mathrm{O}_{2}$ saturation (\%) & $65(7.2)$ & $70.9(7.4)$ & 0.023 \\
\hline Haemoglobin (g/dL) & $14.1(2.1)$ & $13.6(2.2)$ & 0.310 \\
\hline $\begin{array}{l}\text { Arterial } \mathrm{O}_{2} \text { content } \\
\left(\mathrm{mL} \mathrm{O} \mathrm{O}_{2} / 100 \mathrm{~mL}\right)\end{array}$ & $16.6(3.1)$ & $15.4(3.6)$ & 0.086 \\
\hline $\mathrm{O}_{2}$ delivery $(\mathrm{mL} / \mathrm{min}) \dagger$ & $6332(2295)$ & $7263(5337)$ & $0.246 \dagger$ \\
\hline $\begin{array}{l}\text { Pulmonary capacitance } \\
(\mathrm{mL} / \mathrm{mm} \mathrm{Hg}) \ddagger\end{array}$ & $1.28(0.54)$ & $1.64(0.91)$ & 0.013 \\
\hline RV pulsatility & $0.94(0.16)$ & $1.04(0.16)$ & 0.010 \\
\hline Pulse pressure & $44.1(8.9)$ & $40.3(14.1)$ & 0.182 \\
\hline Stroke volume $(\mathrm{mL}) \dagger$ & $51.8(8.7)$ & $61.8(21.7)$ & $0.031 \dagger$ \\
\hline Stroke volume index & $29.2(6.7)$ & $33(7.3)$ & 0.037 \\
\hline Systolic blood pressuret & $125(25)$ & $109(13)$ & $0.028 t$ \\
\hline Mean arterial pressure & $88.6(15.8)$ & $84.8(9.4)$ & 0.278 \\
\hline HR (beats/min) & $79(9.9)$ & $80(11.8)$ & 0.490 \\
\hline Rs (dyn s/cm ${ }^{5}$ ) & $1575(487)$ & $1306(357)$ & 0.015 \\
\hline PVR/SVR & $0.46(0.13)$ & $0.39(0.15)$ & 0.060 \\
\hline TPG & $34.7(8.7)$ & $28.5(10.3)$ & 0.014 \\
\hline BNP $(p g / m L)$ & 558 (859) & $228(340)$ & $0.004 \dagger$ \\
\hline
\end{tabular}

*Paired $t$ test $p$ value presented, except when Wilcoxon signed rank indicated. tData are non-normally distributed; median (IQR) and Wilcoxon signed rank $p$ value presented.

¥Pulmonary capacitance $=$ stroke volume/pulse pressure.

BNP, brain natriuretic peptide; PVR, pulmonary vascular resistance; RV, right ventricular; SVR, systemic vascular resistance; TPG, transpulmonary gradient.

$(p=0.037)$, and improved RV systolic function $(p=0.006)$. In parallel with haemodynamic and echocardiographic evidence of RV unloading, BNP levels fell significantly in subjects at 12-week follow-up (table 4).

\section{Patient status}

Of the 15 patients, 8 were actively listed for LT (figure 1). Of the 7 patients not offered LT, 5 died (mean \pm SD) $504 \pm$ 295 days after treprostinil initiation, while 2 patients remained alive 1059 and 1401 days after treprostinil initiation. After active listing, 7 patients were successfully bridged to LT which occurred at a median of 268 days (range 140-1379 days) after the baseline RHC and treprostinil initiation. The remaining listed patient died 272 days after treprostinil initiation.

\section{DISCUSSION}

The purpose of this investigation was to assess the effects of chronic parenteral treprostinil administration on right heart haemodynamics and echocardiographic function in a PF population referred for LT with an advanced $\mathrm{PH}$ phenotype, characterised by significantly increased PVR and RV dysfunction. ${ }^{10}$ We studied this population either as a bridge to LT or to achieve clinical stabilisation in otherwise transplant ineligible patients at risk of clinical deterioration due to advanced $\mathrm{PH}$ and right heart dysfunction. $^{5} 7{ }^{10}$ Significant improvements were demonstrated in right heart haemodynamics and echocardiographic function in response to chronic parenteral treprostinil infusion, without significant decrement in peripheral oxygen saturation, arterial oxygen content, or oxygen delivery.

At baseline, our subjects had a markedly elevated PVR, moderate to severe RV dysfunction, and thus abnormal coupling between the RV and pulmonary vascular load. In the context of significant RV-pulmonary artery uncoupling, RV afterload reduction leads to a predictable, afterload-dependent improvement in right heart function, as was seen in our cohort by way of improved haemodynamics and echocardiographic parameters. ${ }^{11}$ These improvements in RV afterload and RV function (enhanced RV coupling) likely augmented the circulatory reserve of our patients, explaining their functional advantage and decreased dyspnoea.

The potential for worsening gas exchange with $\mathrm{PH}$-targeted therapy deserves particular attention. We did not appreciate any significant hypoxaemia as assessed by PPO after treprostinil therapy, either at rest or after 6MWD testing. Importantly, pulmonary function (ie, degree of $\mathrm{PF}$ ) remained unaltered during the study and did not likely confound these findings. Although arterial blood gases (ABG) were not obtained to confirm this finding, we can suggest a rationale based on the available literature. Prior work using multiple inert gas elimination technique (MIGET) has demonstrated a relatively preserved V-Q spectrum at rest, manifesting absent or mild resting hypoxaemia in patients with either WHO Group I pulmonary arterial hypertension $(\mathrm{PAH})^{12}$ or isolated $\mathrm{PF}^{13}$ Importantly, during exercise, the MIGET-derived V-Q spectrum remains preserved in both conditions, despite predictable widening of the alveolar-arterial gradient and hypoxaemia. ${ }^{13-15}$ In $\mathrm{PAH}$, this hypoxaemia is driven by a low mixed venous $\mathrm{pO}_{2},{ }^{14}$ while in $\mathrm{PF}$, hypoxaemia is characterised by a relative augmentation in diffusion abnormality, which in turn is further accentuated by low mixed venous oxygenation. ${ }^{16}$ Consequently, a $\mathrm{PH}$-targeted therapy that augments mixed venous oxygenation may be particularly desirable in a cohort of patients with PF and advanced PH to attenuate any predisposition for hypoxaemia at rest or during exercise.
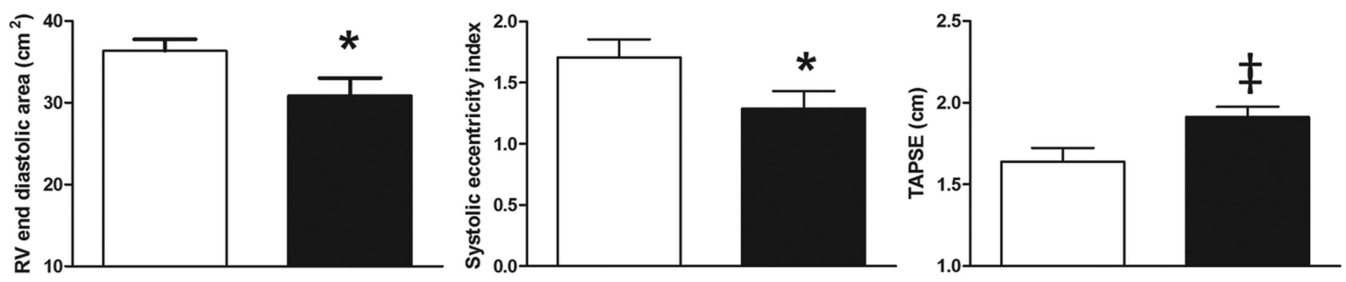

Figure 3 Bar graphs comparing indices of right ventricular size (RV end diastolic area), interventricular septal position (systolic eccentricity index), and RV systolic function (tricuspid annular planar systolic excursion) at baseline (open bars; $n=15$ ) and following 12 weeks of parenteral treprostinil (solid black bars; $n=14)$, with error bars indicating SE. Paired t test results indicate significant improvements in RV size $(p=0.021)$, interventricular septal flattening $(p=0.037)$, and RV systolic function $(p=0.006)$ indices from baseline to week 12 . TAPSE, tricuspid annular planar systolic excursion. 
The use of acute ${ }^{15}$ or chronic ${ }^{17-20} \mathrm{PH}$-targeted, non-prostanoid therapy does not appear to alter gas exchange in patients with $\mathrm{PF}$ without advanced PH during rest or exercise, which challenges the notion of predictable V-Q inequality and hypoxaemia as a direct result of underlying fibrotic lung disease. Despite this finding, acute parenteral prostanoid administration in patients with PF and advanced $\mathrm{PH}$ raises concern for predictable intrapulmonary shunt and hypoxaemia. ${ }^{3} 21$ Interestingly, even patients with PAH (WHO Group I) demonstrated increased shunt by MIGET during acute parenteral prostanoid administration. $^{22}$ The question is whether this potential shunt and hypoxaemia are related to the parenteral route of administration or to the aggressive uptitration strategy routinely implemented when initiating parenteral prostanoid therapy. ${ }^{23}$ In fact, prior studies evaluating acute parenteral prostanoid administration in $\mathrm{PAH}^{22}$ and $\mathrm{PF}$ associated with advanced $\mathrm{PH}^{3}{ }^{21}$ employed this same aggressive uptitration protocol and report relatively worsened gas exchange, V-Q spectrum, and systemic haemodynamic data specifically at the prostanoid dose associated with intolerable adverse reaction and/or unacceptable haemodynamic deterioration. Similar and predictable V-Q inequality has also been demonstrated with other aggressively titrated, non-prostanoid vasodilators in patients with $\mathrm{PAH} .^{24}$

This approach may result in undesired physiology driven by decreased SVR, including reflex tachycardia, increased cardiac output (CO), systemic hypotension, and unchanged or increased PVR/SVR ratio. Comparable untoward physiology was recently demonstrated after riociguat therapy in patients with $\mathrm{PF}$ and advanced $\mathrm{PH}$ with resultant mild hypoxaemia. ${ }^{25}$ While a patient with $\mathrm{PAH}$ may be able to tolerate an aggressive uptitration of parenteral prostanoid, ${ }^{22}$ this strategy may place a patient with $\mathrm{PF}$ and advanced PH at risk of acute cardiopulmonary decompensation. ${ }^{3}$ Consequently, a more gradual parenteral prostanoid uptitration approach may attenuate shunt physiology and subsequent hypoxaemia, especially if significant systemic vasodilation and the resulting abrupt rise in $\mathrm{CO}$ are avoided. Cardiac output may itself be associated with increased intrapulmonary shunt. ${ }^{26}$ Based on the above, we surmise that a gentler uptitration of parenteral prostanoid, as employed in our study PF population with advanced $\mathrm{PH}$, may lessen the potential for haemodynamic instability and/or hypoxaemia and rather parallel the chronic parenteral prostanoid administration haemodynamic and gas exchange data reported in WHO Group I PAH.

The inclusion of patients restricted to a severe baseline $\mathrm{PH}$ phenotype likely decreased the predisposition to arterial oxygen desaturation in response to treprostinil. Our subjects had a markedly elevated PVR and borderline reduced CO at baseline with evidence of significantly improved right heart function following treprostinil infusion, delineated by less septal bowing, a falling right atrial pressure, and improved CO. These salutary effects on right heart function may further optimise arterial oxygen content via enhanced mixed venous oxygen saturation. ${ }^{16} 27$

The improvement of $59 \mathrm{~m}$ in 6MWD following 12 weeks of parenteral treprostinil was noted in parallel with improvements in the UCSD SOB questionnaire and the SF-36 MCS, representing preliminary but encouraging findings. Recent independent studies in subjects with idiopathic pulmonary fibrosis (IPF) and Group I PAH suggested the minimally clinically important significant 6MWD difference to be $24-45 \mathrm{~m}$ and $25-38 \mathrm{~m}$, respectively. ${ }^{28} 29$ The augmentation in functional capacity seen in our subjects with PF-PH in response to treprostinil does not contravene prior studies reporting a lack of improvement in functional parameters in patients with PF treated with PH-targeted therapies. ${ }^{17-19}$ Patients in these prior studies had no or mild $\mathrm{PH}$, while our subjects had an average mPAP $>45 \mathrm{~mm} \mathrm{Hg}$, a markedly elevated PVR, and degrees of $\mathrm{RV}$ dilatation and dysfunction comparable to severe WHO Group I PAH. ${ }^{30}$

These physiological differences are likely critical when considering the potential response to $\mathrm{PH}$-targeted therapies, given that patients with advanced lung disease in the absence of advanced $\mathrm{PH}$ typically do not possess evidence of a circulatory limitation to exercise. ${ }^{31} 32$ In contrast, patients with parenchymal lung disease (COPD or PF) and advanced $\mathrm{PH}$ demonstrate (in addition to their inherent ventilatory limitation) a circulatory limitation on exertion and an overall cardiopulmonary exercise stress test profile similar to isolated Group I PAH, with blunted oxygen pulse and marked ventilatory inefficiency (ie, increased $\left.\mathrm{V}_{\mathrm{E}} / \mathrm{V}_{\mathrm{CO} 2}\right) .{ }^{31}{ }^{32}$ Importantly, the moderate degree of restrictive lung disease in our patient cohort may not have warranted consideration of LT, had it been isolated from the severe extent of superimposed $\mathrm{PH}$. Interestingly, a recent post hoc analysis of a placebo-controlled randomised clinical trial in $\operatorname{IPF}^{20}$ showed that the subgroup of patients with, compared with those without, RV dysfunction and RV hypertrophy improved their 6MWD in response to sildenafil. ${ }^{33}$ In addition, the Royal Brompton group retrospectively reported a significant 6MWD improvement with sildenafil in a mixed interstitial lung disease population with pulmonary function and right heart haemodynamics similar to our experience. ${ }^{2}$

The combined observations of improved right heart function and stable arterial oxygen saturation in our PF-PH cohort after chronic parenteral treprostinil suggests the advanced $\mathrm{PH}$ phenotype may be critical when considering PH-targeted therapy, as it lends itself towards an increased likelihood of improved circulatory reserve and decreased risk of hypoxaemia. As such, an advanced $\mathrm{PH}$ phenotype in the context of chronic respiratory disease may be essential for predicting a beneficial response and minimising potential adverse effects of therapy.

\section{LIMITATIONS}

Limitations of this study include the heterogeneity of the PF population, variable background $\mathrm{PH}$-targeted therapy, and the absence of ABG testing. The absence of a placebo arm is a particularly significant limitation; therefore, our findings must be confirmed with a randomised, placebo-controlled trial. At this point, the routine use of $\mathrm{PH}$-targeted therapy in $\mathrm{PF}-\mathrm{PH}$ is not recommended and should only be cautiously considered at specialised PH centres to avoid the serious potential for worsening cardiopulmonary status in this patient population. In addition, the explanation proposed for the lack of significant hypoxaemia with parenteral prostanoid in our PF-PH cohort remains speculative and requires further investigation. To address the limitation of multiple comparisons in the domains of pulmonary function, quality of life, and haemodynamics, we calculated that observed $\mathrm{p}$ values $<0.05$ corresponded to a maximum q-value of 0.067 , indicating that the proportion of significant findings attributable to false discovery is small. As such, we were reassured to see encouraging results in a real-world cohort of patients with PF referred for LT, characterised by an 'advanced $\mathrm{PH}$ and right heart dysfunction' phenotype. The lack of ABG testing is offset by stable arterial oxygen content, oxygen delivery, and oxygen saturation values at rest and 6MWD testing, following treprostinil therapy.

\section{CONCLUSION}

This open-label study suggests that gradual initiation and chronic administration of parenteral treprostinil therapy may 
improve haemodynamics and right heart function without compromising systemic oxygenation in an advanced $\mathrm{PH}$ phenotype with RV dysfunction in the setting of PF. These findings are only hypothesis generating and require confirmation in a multicentre, randomised study design. Future studies of $\mathrm{PH}$-targeted therapy for PF should focus on patients with PF with the combination of advanced $\mathrm{PH}$ and RV dysfunction, as these subjects may have greater capacity for benefit. Finally, given the high mortality inherent to this population, a future study may consider survival as an endpoint.

\section{Author affiliations}

${ }^{1}$ Thoracic Transplantation, Heart-Lung Institute, St Joseph Hospital \& Medical Center, Phoenix, Arizona, USA

${ }^{2}$ Division of Rheumatology, Department of Medicine, University of Michigan, Ann Arbor, Michigan, USA

${ }^{3}$ Cardiovascular Division, Department of Medicine, Perelman School of Medicine at the University of Pennsylvania, Philadelphia, Pennsylvania, USA

${ }^{4}$ Division of Pulmonary and Critical Care Medicine, Department of Medicine, David Geffen School of Medicine at UCLA, Los Angeles, California, USA

${ }^{5}$ Department of Medicine Statistics Core, David Geffen School of Medicine at UCLA, Los Angeles, California, USA

${ }^{6}$ George Washington University, School of Medicine and Health Sciences,

Washington, DC, USA

${ }^{7}$ Division of Radiology, Department of Medicine, David Geffen School of Medicine at UCLA, Los Angeles, California, USA

${ }^{8}$ Cardiovascular Division, Temple University School of Medicine, Philadelphia, Pennsylvania, USA

Acknowledgements The authors would like to acknowledge Lynne Yoder RN, Bryant Torres BS, Glenna Traiger RN, Eileen Callahan CCRP, and Paul Lopez LVN for their dedicated support with data collection and study coordination as well as NHLBI HL112990 to JAB.

Contributors RS: conceptual design; patient care and procedures; writing of manuscript; data collection. DK: conceptual design; editing of manuscript; statistical support. AV: blinded review of echocardiograms. AD: patient care and procedures; statistical support. PM: statistical support. ED: statistical support. JAB: patient care and procedures. SSW: patient care and procedures. SD: patient care and procedures; data collection. SSS: patient care. JGG: blinded review of chest CT scans. FA: blinded review of chest CT scans. JPLIII: conceptual design; patient care. DJR: patient care. PRF: editing of manuscript; blinded review of echocardiograms. RS: conceptual design; patient care and procedures; writing of manuscript; data collection.

Funding NHLBI and United Therapeutics.

Competing interests None.

Ethics approval Institutional Review Board at University of California, Los Angeles.

Provenance and peer review Not commissioned; externally peer reviewed.

\section{REFERENCES}

1 Minai OA, Sahoo D, Chapman JT, et al. Vaso-active therapy can improve 6-min walk distance in patients with pulmonary hypertension and fibrotic interstitial lung disease. Respir Med. 2008;102:1015-20.

2 Corte TJ, Gatzoulis MA, Parfitt L, et al. The use of sildenafil to treat pulmonary hypertension associated with interstitial lung disease. Respirology 2010;15:1226-32.

3 Olschewski H, Ghofrani HA, Walmrath D, et al. Inhaled prostacyclin and iloprost in severe pulmonary hypertension secondary to lung fibrosis. Am J Respir Crit Care Med 1999;160:600-7.

4 Le Pavec J, Girgis RE, Lechtzin N, et al. Systemic sclerosis-related pulmonary hypertension associated with interstitial lung disease: impact of pulmonary arterial hypertension therapies. Arthritis Rheum 2011;63:2456-64.

5 Saggar R, Shapiro SS, Ross DJ, et al. Treprostinil to reverse pulmonary hypertension associated with idiopathic pulmonary fibrosis as a bridge to single-lung transplantation. J Heart Lung Transplant 2009;28:964-7.

6 Cottin V, Le Pavec J, Prevot G, et al. Pulmonary hypertension in patients with combined pulmonary fibrosis and emphysema syndrome. Eur Respir J 2010:35:105-11.
7 McLaughlin VV, Archer SL, Badesch DB, et al. ACCF/AHA 2009 expert consensus document on pulmonary hypertension: a report of the American College of Cardiology Foundation Task Force on Expert Consensus Documents and the American Heart Association: developed in collaboration with the American College of Chest Physicians, American Thoracic Society, Inc., and the Pulmonary Hypertension Association. Circulation 2009;119:2250-94.

8 American Thoracic Society Statement. Guideline for the six-minute walk test. Am J Respir Crit Care Med 2002;166:111-17.

9 Mathier MA, McDevitt S, Saggar R. Subcutaneous treprostinil in pulmonary arterial hypertension: practical considerations. J Heart Lung Transplant 2010;29: 1210-17.

10 Hoeper MM, Andreas S, Bastian A, et al. Pulmonary hypertension due to chronic lung disease: updated Recommendations of the Cologne Consensus Conference 2011. Int J Cardiol 154(Suppl 1):S45-53.

11 Van de Veerdonk MC, Kind T, Marcus JT, et al. Progressive right ventricular dysfunction in patients with pulmonary arterial hypertension responding to therapy. J Am Coll Cardiol 2011;58:2511-19.

12 Dantzker DR, Bower JS. Mechanisms of gas exchange abnormality in patients with chronic obliterative pulmonary vascular disease. J Clin Invest 1979;64:1050-5.

13 Agusti AG, Roca J, Gea J, et al. Mechanisms of gas-exchange impairment in idiopathic pulmonary fibrosis. Am Rev Respir Dis 1991;143:219-25.

14 Dantzker DR, D'Alonzo GE, Bower JS, et al. Pulmonary gas exchange during exercise in patients with chronic obliterative pulmonary hypertension. Am Rev Respir Dis 1984;130:412-16.

15 Blanco I, Ribas J, Xaubet A, et al. Effects of inhaled nitric oxide at rest and during exercise in idiopathic pulmonary fibrosis. J Appl Physiol 2011;110:638-45.

16 Wagner PD. Influence of mixed venous PO2 on diffusion of 02 across the pulmonary blood:gas barrier. Clin Physiol 1982;2:105-15.

17 King TE Jr, Brown KK, Raghu G, et al. BUILD-3: a randomized, controlled trial of bosentan in idiopathic pulmonary fibrosis. Am J Respir Crit Care Med 2011;184: 92-9.

18 Raghu G, Behr J, Brown KK, et al. Treatment of idiopathic pulmonary fibrosis with ambrisentan: a parallel, randomized trial. Ann Intern Med 2013;158:641-9.

19 Raghu G, Million-Rousseau R, Morganti A, et al. Macitentan for the treatment of idiopathic pulmonary fibrosis: the randomised controlled MUSIC trial. Eur Respir J 2013:42:1622-32.

20 Zisman DA, Schwarz M, Anstrom KJ, et al. A controlled trial of sildenafil in advanced idiopathic pulmonary fibrosis. N Engl J Med 2010;363:620-8.

21 Ghofrani HA, Wiedemann R, Rose F, et al. Sildenafil for treatment of lung fibrosis and pulmonary hypertension: a randomised controlled trial. Lancet 2002:360:895-900.

22 Bratel T, Lagerstrand L, Brodin LA, et al. Ventilation-perfusion relationships in pulmonary arterial hypertension: effect of intravenous and inhaled prostacyclin treatment. Respir Physiol Neurobiol 2007;158:59-69.

23 Barst RJ, Rubin LJ, Long WA, et al. A comparison of continuous intravenous epoprostenol (prostacyclin) with conventional therapy for primary pulmonary hypertension. N Engl J Med 1996;334:296-301.

24 Dantzker DR, Bower JS. Pulmonary vascular tone improves VA/Q matching in obliterative pulmonary hypertension. J App/ Physiol 1981;51:607-13.

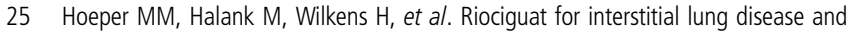
pulmonary hypertension: a pilot trial. Eur Respir J 41:853-60.

26 Lynch JP, Mhyre JG, Dantzker DR. Influence of cardiac output on intrapulmonary shunt. J Appl Physiol 1979;46:315-21.

27 Agusti AG, Rodriguez-Roisin R. Effect of pulmonary hypertension on gas exchange. Eur Respir J. 1993;6:1371-7.

28 du Bois RM, Weycker D, Albera C, et al. Six-minute-walk test in idiopathic pulmonary fibrosis: test validation and minimal clinically important difference. Am J Respir Crit Care Med 2011;183:1231-7.

29 Mathai SC, Puhan MA, Lam D, et al. The minimal important difference in the 6-minute walk test for patients with pulmonary arterial hypertension. Am J Respir Crit Care Med 2012;186:428-33.

30 Forfia PR, Fisher MR, Mathai SC, et al. Tricuspid annular displacement predicts survival in pulmonary hypertension. Am J Respir Crit Care Med 2006;174: 1034-41.

31 Boutou AK, Pitsiou GG, Trigonis I, et al. Exercise capacity in idiopathic pulmonary fibrosis: the effect of pulmonary hypertension. Respirology 2011;16:451-8.

32 Glaser S, Noga O, Koch B, et al. Impact of pulmonary hypertension on gas exchange and exercise capacity in patients with pulmonary fibrosis. Respir Med 2009:103:317-24.

33 Han MK, Bach DS, Hagan PG, et al. Sildenafil preserves exercise capacity in patients with idiopathic pulmonary fibrosis and right-sided ventricular dysfunction. Chest 2013;143:1699-1708. 